\title{
Sliding Mode Control for Multiagent System with Time-Delay and Uncertainties: An LMI Approach
}

\author{
Pengfei Guo, ${ }^{1}$ Jie Zhang, ${ }^{1}$ Ming Lyu, ${ }^{2}$ and Yuming Bo ${ }^{1}$ \\ ${ }^{1}$ School of Automation, Nanjing University of Science \& Technology, Nanjing 210094, China \\ ${ }^{2}$ Information Overall Department, North Information Control Group Co., Ltd., Nanjing 211153, China \\ Correspondence should be addressed to Jie Zhang; zhangjie.njust@gmail.com
}

Received 24 July 2013; Accepted 13 September 2013

Academic Editor: Rongni Yang

Copyright (c) 2013 Pengfei Guo et al. This is an open access article distributed under the Creative Commons Attribution License, which permits unrestricted use, distribution, and reproduction in any medium, provided the original work is properly cited.

\begin{abstract}
This paper considers the sliding mode control of multiagent systems (MAS) with time-delay and uncertainties in terms of linear matrix inequality (LMI). By constructing virtual feedback control method, the design of control system is simplified for time-delay independent system without uncertainties. For a class of uncertain systems with single time-delay, the essence of SMC design is analyzed in order to acquire a simple method for designing sliding surface. In terms of multiple timedelay system with uncertainties, a sufficient condition for sliding surface with independent time-delay is acquired, while control law is also designed to ensure the robust stability of closed-loop system. Finally, the effectiveness of conclusion is demonstrated by simulation results.
\end{abstract}

\section{Introduction}

Research on application of multiagent system (MAS) began in the middle of 1980s with a significant growth trend in recent years. Multiagent technology has been one of the hot issues studied in the field of artificial intelligence presently. Multiagent system, as a frontier discipline in artificial intelligence and an important branch of distributed artificial intelligence research, aims to build large and complicated system into small, mutually communicated, and coordinated system that is easy for management. Research on multipleagent is involving the knowledge, objective, skills, planning as well as how to coordinate between agents, and so forth [1].

However, as the structure of MAS is growing increasingly complicated, more and more problems appear in this field, such as the basic problem of time-delay, random packet loss, uncertainty of system model, and influence of unknown interference on system, all of which can result in interference on the communication of MAS or signal channel congestion. Sliding mode control (SMC) enables a system to switch between two control laws by making use of the discontinuity of control, accordingly generating a new movement having no direct relationship with the original system: sliding mode. Such movement mainly has two features: Firstly, appropriate sliding surface can be selected to achieve dynamic feature of the system so as to meet the demand in performance indicators of closed-loop system. Secondly, closed-loop system reveals insensitivity to uncertainty or random interference. Such invariance proves that sliding mode control is particularly appropriate to be used as robust controller for an uncertain system. Therefore, more and more scholars pay attention to the application of SMC in MAS and relevant research [2-4].

Actually, system usually has uncertainties and varying parameters, while mathematical description is always full of uncertainties and influenced by the disturbance from exterior. In particular, a system usually contains complicated mathematical description which can be viewed as the perturbation of system, whereas these uncertain factors usually fail to completely meet the matching criteria of the system. Usually the original system will be changed to simple form of sliding mode through nonsingular state transformation, then Lyapunov equation of reduced order will be constructed to acquire the sufficient condition of Riccati equation of the designed sliding surface [5-7]. With the development of robust control theory in recent years, particularly the development of linear matrix inequality, many scholars begin to focus on this field [8]. For the 
uncertain system which meets the matching requirements, Choi designed a new sliding surface, by using quadratic stability theory, acquired a sufficient condition based on LMI, and described the approaching process of SMC [9]. Reference [10] concerned SMC of switched systems with stochastic Perturbation, derived a sufficient condition for the existence of reduced-order sliding mode dynamics. In [11], local stability theory was used to acquire the necessary and sufficient condition for general nonmatching linear SMC system with uncertainties, and then guaranteed the global stability of the system. Reference [6] carried out singular value decomposition of the input matrix and changed the uncertain linear system into simple form through nonsingular state transformation. Moreover, through construction of Lyapunov equation, sliding surface was designed to provide the sufficient condition of system stability in the form of Riccati equation. In [12], through analyzing the features of the uncertain parts in the system after nonsingular transformation, a new system matching condition was provided additionally with a new method for constructing sliding surface, at the same time chattering of the system was well suppressed. References $[13,14]$ carried out further researches on the design of sliding surface. For a class of singular stochastic system with Markovian switching, [15] designed an integral sliding surface function, by introducing some specified matrices, a new sufficient condition is proposed in terms of strict linear matrix inequality, which guaranteed the stochastic stability of the sliding mode dynamics. Reference [16] designed an observer to estimate the system states, and a sliding-mode control scheme is synthesized for the reaching motion based on the state estimates.

The general communication delay in MAS has always been the focus of scholars. In recent years, with the development of robust control theory, research on the stability of time-delay system and the design of controller has achieved great progress. References $[17,18]$ presented a common timedelay model, concluded the condition of time-delay system stability, and introduced the design method of state feedbackbased controller. Targeting at time-delay dependence model, the literature [19-21] discussed the conditions of stability and analyzed the influence of time-delay transformation model and Lyapunov-Krasovskii function on time-delay system stability. The literature [22] presented a new time-delay transformed model and corresponding Lyapunov-Krasovskii function, yet as the result by Lyapunov-Krasovskii was relatively conservative, the stability condition is conservative and thereby did not fit fast transformed system. In 2001, Fridman put forward a singular time-delay state transformation model and the corresponding Lyapunov-Krasovskii function [2325 ], which is less conservative and suitable to fast transformation time-delay systems, while the design method of stabilizing controller was also introduced.

In this paper, multiagent models under three different conditions are studied. First of all, time-delay independent system without uncertainties is investigated, and sliding mode of quadratic stability is constructed by making use of virtual state feedback. Then, uncertain system with single delay is studied, and nonsingular transformation design is applied to make the system reach quadratic stability. In addition, through quadratic stability-based design of robust sliding surface for time-delay independent system with nonmatching uncertainties, we acquire sufficient condition of quadratic stable surface for reduced order system, and controller is designed for the approaching motions of multiple time-delay system. Both approaching and sliding motion show a desirable robust to nonmatching uncertainties and exterior interference. Finally, three illustrative examples show the effectiveness of proposed theorems.

\section{Problem Formulation}

Concerning a type of MAS, as shown in Figure 1, information flow is transmitted among all agents directly. Let $G=\{v, e\}$ be a directed graph of order $n$ with the set of agents $v=$ $\{1,2, \ldots, n\}$ and set of edges $e \subseteq v \times v$; edge $(i, j) \in e$ indicates that agent $j$ is able to receive information from agent $i$. The adjacent matrix $A=\left[a_{i j}\right] \in R^{n \times n}$ of directed graph $G$ is defined as follows: if information exchange exists between two agents $i$ and $j$, then $a_{i j}$ denotes the throughput from $i$ to $j$. At the same time, agent $i$ may also receive information from $j$, yet $a_{i j}$ is not necessarily equal to $a_{j i}$. Taking into account that if the MAS is static, the connection structure inside the system is not varying with time. In other words, the system is fixed and contains no uncertain items; yet communication delay exists among all agents; then the system model can be summarized as a time-delay independent system without uncertainties, and the model is given as follows:

$$
\begin{gathered}
\dot{x}(t)=A x(t)+A_{d} x(t-\tau)+B(u(t)+f(t)), \\
x(t)=\Psi(t) \quad t \in[-\tau, 0],
\end{gathered}
$$

where $x(t) \in R^{n}$ is system state, $u(t) \in R^{m}$ denotes system control input, $f(t)$ is disturbance, $A, A_{d}(t)$, and $B$ are constant matrices with appropriate dimensions.

However, in practical system, multiagents may be in a dynamic state. Communication connection among agents within communication range may be interrupted as the distance increases; yet agents beyond communication range may also establish connection with each other through movement; this uncertain factor is one of the major reasons resulting in system structure uncertainty. If the state variation of a specific agent in the system is only related to its own state, then the system model is defined as uncertain system with single timedelay, and the model is described as follows:

$$
\begin{gathered}
\dot{x}(t)=(A+\Delta A(t)) x(t)+\left(A_{d}+\Delta A_{d}(t)\right) x(t-\tau) \\
+B(u(t)+f(t)), \\
x(t)=\Psi(t) \quad t \in[-\tau, 0],
\end{gathered}
$$

where $x(t) \in R^{n}$ denotes system state, $u(t) \in R^{m}$ is system control input, $\Delta A(t)$ and $\Delta A_{d}(t)$ are internal parameter perturbation arising from uncertain factors, $f(t)$ denotes external disturbance, and $A, A_{d}(t)$, and $B$ are constant matrices with appropriate dimensions.

If an agent in MAS is influenced by multiple time-delays, then the system model should be a multiple time-delay 


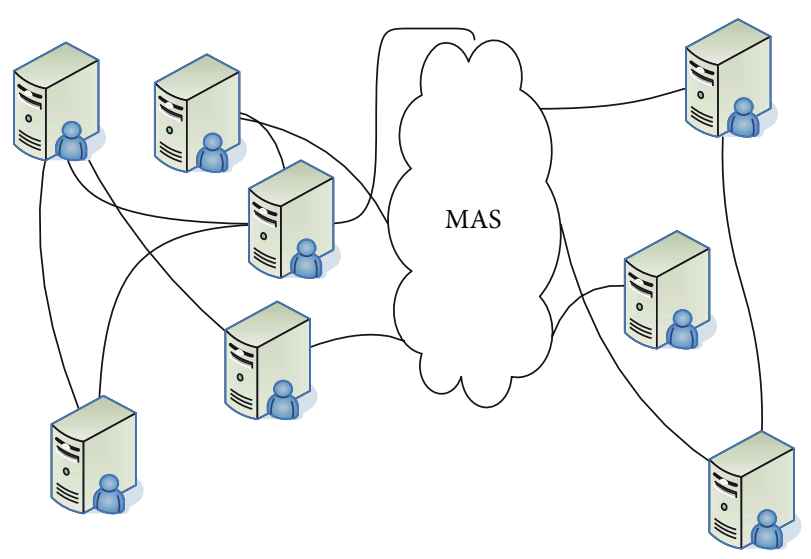

FIGURE 1: MAS with an fixed structure and time-delay.

system with uncertainties, and the model is described as follows:

$$
\begin{gathered}
\dot{x}(t)=(A+\Delta A(t)) x(t)+\sum_{i=1}^{N}\left(A_{d}+\Delta A_{d}(t)\right) x_{i}\left(t-\tau_{i}\right) \\
+B(u(t)+f(t)), \\
x(t)=\Psi(t) \quad t \in[-\tau, 0],
\end{gathered}
$$

where $x(t) \in R^{n}$ denotes system state, $u(t) \in R^{m}$ is system control input, $\Delta A(t)$ and $\Delta A_{d}(t)$ are internal parameter perturbation arising from uncertain factors, $f(t)$ denotes external disturbance, $A, A_{d}(t)$, and $B$ are constant matrices with appropriate dimensions, and $N$ is the number of agents in the system. In both cases, the system is evolved into one shown in Figure 2. Since the MAS may be in a dynamic state or affected by external connection interference, the communication connection among multiagents features uncertain factors, and the dashed line indicates disconnection or connection occurring at any time.

For the systems shown in (1), (2), and (3), we will use the following assumptions:

Assumption 1. Supposing that $(A, B)$ could be stabilized, then there is a matrix $K$ makes $\bar{A}=A-B K$ stabilizing and $\operatorname{rank}(B)=m \leq n$.

Assumption 2. The disturbance has an upper bound, which meets $\|f(t)\| \leq \delta_{f}$.

Assumption 3. Time-delay $\tau_{i}$ is boundary, and $\left\|\tau_{i}\right\|_{i \in N} \leq \tau$.

Assumption 4. The perturbation parameter of the system satisfies

$$
\left[\begin{array}{ll}
\Delta A & \Delta A_{d}
\end{array}\right]=G D(t)\left[\begin{array}{ll}
H & H_{d}
\end{array}\right] .
$$

Respectively, $G, H$, and $H_{d}$ are known constant matrices, $D(t)$ is time-delay uncertain matrix, yet Lebesguemeasurable, and $D^{T}(t) D(t) \leq I$.

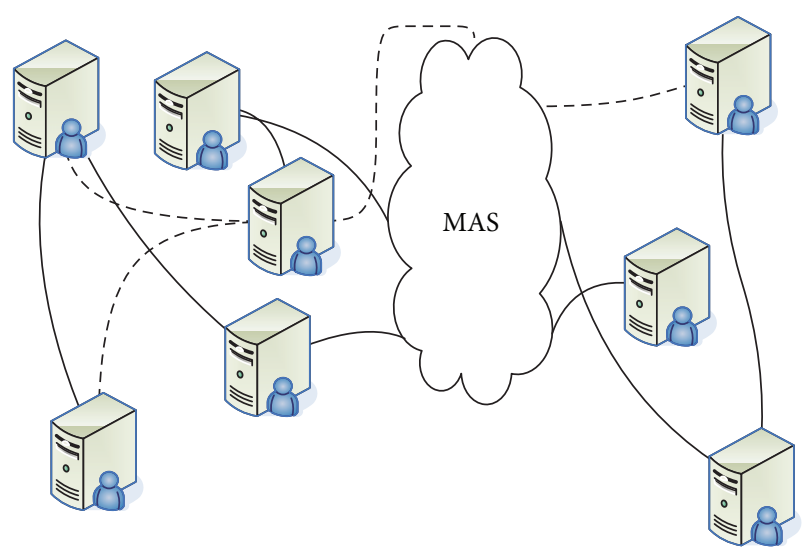

FIGURE 2: MAS with an unfixed structure and time-delay.

For the design of SMC, usually it consists of two parts. Firstly, design a stable sliding surface which allows time-delay system to maintain a good dynamic performance under internal and external parameter perturbation. Secondly, choose proper control law so as to guarantee the system to meet the sliding mode condition from any states, that is, to reach the sliding surface in finite time.

Besides, a few frequently used and important lemmas are presented here.

Lemma 5. Let $Y=Y^{T}, D, E$, and $F(t)$ be real matrix of proper dimensions, and $F^{T}(t) F(t) \leq I$, then inequality $Y+D F E+$ $(D F E)^{T}<0$ holds if there exists a constant $\varepsilon$, which makes the following equation hold

$$
Y+\varepsilon D D^{T}+\varepsilon^{-1} E^{T} E<0 .
$$

Lemma 6 (Schur complement). Given a symmetric matrix $S=\left[\begin{array}{ll}S_{11} & S_{12} \\ S_{21} & S_{22}\end{array}\right]$, where $S_{11}$ is $r \times r$ dimensional, the following three conditions are equivalent:
(1) $S<0$;
(2) $S_{11}<0, S_{22}-S_{12}^{T} S_{11}^{-1} S_{12}<0$;
(3) $S_{22}<0, S_{11}-S_{12} S_{22}^{-1} S_{12}^{T}<0$.

\section{Main Results}

3.1. Time-Delay Independent System without Uncertainties. In this section, the control law and sliding surface design of MAS as shown in Figure 1 are investigated.

Theorem 7. For system (1) which meets Assumptions 1 and 2, then the system from any initial states will meet the sliding mode reaching condition; that is, it will gradually be driven onto the sliding surface $S(t)=B^{T} P x(t)$ within finite time with control law

$$
u(t)=u_{e q}(t)+u_{s w}(t),
$$

where the equivalent control is $u_{e q}(t)=-\left(B^{T} P B\right)^{-1}\left[B^{T} P A x(t)\right.$ $\left.+B^{T} P A_{d} x(t-\tau)\right]$ and nonlinear switch control is $u_{s w}(t)=$ 
$-\left(B^{T} P B\right)^{-1}\left[\left|B^{T} P B\right| \delta_{f}+\varepsilon\right] \operatorname{sgn}(S)$, in which $\varepsilon$ is a positive constant.

Proof. Choose a Lyapunov function as

$$
V=\frac{1}{2} S^{T} S
$$

Apparently, for all $S(x, t) \neq 0$, Lyapunov function $V$ is positive-definite; it follows that the Lyapunov derivative corresponding to (1), then

$$
\begin{aligned}
\dot{V}= & S^{T} \dot{S} \\
\dot{S}= & B^{T} P \dot{x} \\
= & B^{T} P A x(t)+B^{T} P A_{d} x(t-\tau) \\
& +B^{T} P B u(t)+B^{T} P B f(t) \\
= & B^{T} P B u_{\mathrm{sw}}(t)+B^{T} P B f(t) \\
\leq & -\varepsilon \operatorname{sgn}(S) .
\end{aligned}
$$

So there is

$$
\dot{S}=S^{T} S \leq-\varepsilon|S| \leq 0 .
$$

This proves that system from any initial states meets the sliding mode reaching condition, thus the proof of Theorem 7 is complete.

Next, the sliding surface with robust stability will be designed. The control law is modified into the form of virtual feedback, ensuring the sliding mode control system with good robust in time-delay condition.

Theorem 8. The system (1) is quadratically stable if there exists symmetric positive-definite matrices $X$ and $V$ and general matrix L of appropriate dimensions, such that is shown in (10); moreover, the sliding surface of $(1)$ is $S(t)=B^{T} P x(t)$, in which $P=X^{-1}$

$$
\left[\begin{array}{ccc}
A X+X A^{T}-B L-L^{T} B^{T} & A_{d} V & X \\
V A_{d}^{T} & -V & 0 \\
X & 0 & -V
\end{array}\right]<0 .
$$

Proof. First, we express (6) as a virtual feedback form

$$
u(t)=-K x(t)+v(t)
$$

where $v(t)=K x(t)+u_{\mathrm{eq}}(t)+u_{\mathrm{sw}}(t)$, substituting (1) with (11), then the whole system is represented as

$$
\dot{x}(t)=\bar{A} x(t)+A_{d} x(t-\tau)+B(v(t)+f(t)),
$$

where $\bar{A}=A-B K$. Choose Lyapunov function as

$$
V(x, t)=x^{T}(t) P x(t)+\int_{t-\tau}^{t} x^{T}(s) Q x(s) d s .
$$

Its derivative along (12) is

$$
\begin{aligned}
\dot{V}= & 2 x^{T} P \dot{x}+x^{T} Q x-x^{T}(t-\tau) Q x(t-\tau) \\
= & 2 x^{T} P\left[\bar{A} x+A_{d} x(t-\tau)+B(v+f)\right] \\
& +x^{T} Q x-x^{T}(t-\tau) Q x(t-\tau) .
\end{aligned}
$$

From Theorem 7, it has been indicated that with control law (6), the sliding mode control system meets the reaching condition; therefore, the system can reach the sliding surface within limited time, in this case $S=B^{T} P x=0$ holds; in addition, $S^{T}=x^{T} P^{T} B=0$ holds; the previous equation can be simplified as

$$
\begin{aligned}
\dot{V}= & 2 x^{T} P \bar{A} x+2 x^{T} P A_{d} x(t-\tau) \\
& +x^{T} Q x-x^{T}(t-\tau) Q x(t-\tau) \\
= & {\left[\begin{array}{ll}
x^{T}(t) & x^{T}(t-\tau)
\end{array}\right] M\left[\begin{array}{c}
x(t) \\
x(t-\tau)
\end{array}\right], }
\end{aligned}
$$

where

$$
M=\left[\begin{array}{cc}
\bar{A}^{T} P+P \bar{A}+Q & P A_{d} \\
A_{d}^{T} P & -Q
\end{array}\right] .
$$

If system (1) is quadratically stable, we have $M<0$. Multiply diag $\left\{P^{-1} I\right\}$ in both sides of (16), then

$$
\left[\begin{array}{cc}
P^{-1} \bar{A}^{T}+\bar{A} P^{-1}+P^{-1} Q P^{-1} & A_{d} \\
A_{d}^{T} & -Q
\end{array}\right]<0 .
$$

Letting $P^{-1}=X$ and $L=K x$, then

$$
\left[\begin{array}{cc}
X A^{T}+A X-B L-L^{T} B^{T}+X Q X & A_{d} \\
A_{d}^{T} & -Q
\end{array}\right]<0 .
$$

From Lemma 6, we have

$$
\left[\begin{array}{ccc}
X A^{T}+A X-B L-L^{T} B^{T} & A_{d} & X \\
A_{d}^{T} & -Q & 0 \\
X & 0 & -Q^{-1}
\end{array}\right]<0 .
$$

Multiply diag $\left\{\begin{array}{lll}I & Q^{-1} & I\end{array}\right\}$ on both sides of (19), then

$$
\left[\begin{array}{ccc}
X A^{T}+A X-B L-L^{T} B^{T} & A_{d} Q^{-1} & X \\
Q^{-1} A_{d}^{T} & -Q^{-1} & 0 \\
X & 0 & -Q^{-1}
\end{array}\right]<0 .
$$

Letting $Q^{-1}=V$, so the proof of Theorem 8 is complete.

Remark 9. Based on Theorems 7 and 8, with the effect of robust control (6), system (1) is quadratically stable. From control law (6) and sliding surface (11), the virtual feedback constructed here simplifies the design of sliding surface. In the design process, nonsingular transformation is not required, accordingly simplifies the design of controller. 
3.2. Uncertain System with Single Time-Delay. Since the MAS structure may change at any time, which could result in parameters perturbation internally. In this section, uncertain system with single time-delay will be discussed, which is shown in (2).

According to assumption, a nonsingular matrix $T$ can be chosen such that $T B=\left[\begin{array}{c}0_{(n-m) \times m} \\ B_{m}\end{array}\right]$, where $B_{m}$ is nonsingular with $\operatorname{rank}\left(B_{m}\right)=m$. According to the definition in [26], let us select $T=\left[\begin{array}{c}U_{2}^{T} \\ U_{1}^{T}\end{array}\right]$, where $U_{1} \in R^{n \times m}$ and $U_{2} \in R^{n \times(n-m)}$ are two unitary matrices resulting from singular value decomposition of matrix $B$, then

$$
B=\left[\begin{array}{ll}
U_{1} & U_{2}
\end{array}\right]\left[\begin{array}{c}
\Sigma \\
0_{(n-m) \times m}
\end{array}\right] V^{T},
$$

where $\Sigma \in R^{m \times m}$ is a symmetric positive-definite matrix, $V \in$ $R^{m \times m}$ is a unitary matrix, by the state transformation $y=T x$, and (2) have the regular form

$$
\begin{gathered}
\dot{y}(t)=(\bar{A}+\Delta \bar{A}(t)) y(t)+\left(\bar{A}_{d}+\Delta \bar{A}_{d}(t)\right) y(t-\tau) \\
+\left[\begin{array}{c}
0_{(n-m) \times m} \\
B_{m}
\end{array}\right](u(t)+f(t)) \\
y(t)=\Phi(t) \quad t \in[-\tau, 0]
\end{gathered}
$$

where $\bar{A}=T A T^{-1}, \bar{A}_{d}=T A_{d} T^{-1}, \Delta \bar{A}(t)=T \Delta A(t) T^{-1}$, $\Delta \bar{A}_{d}(t)=T \Delta A_{d}(t) T^{-1}$, and $\Phi(t)=T \Psi(t)$.

In the following theorem, the design of sliding mode controller is presented. Moreover, the sliding surface can be chosen as

$$
S(t)=G y=C y_{1}+y_{2}=0,
$$

where $G=\left[\begin{array}{ll}C & I\end{array}\right], y=\left[\begin{array}{l}y_{1} \\ y_{2}\end{array}\right]$, and $C \in R^{m \times(n-m)}$.

Theorem 10. For uncertain system with single delay such as (2), which meets Assumptions 2 and 4 and sliding surface is given by (23). Then, the system can be driven onto the sliding surface in limit time with control law

$$
\begin{aligned}
u(t)=-B_{m}^{-1}[ & \Phi S+G(\bar{A}+\Delta \bar{A}) y(t) \\
& \left.+G\left(\bar{A}_{d}+\Delta \bar{A}_{d}\right) y(t-\tau)+B_{m} \delta_{f}\right] .
\end{aligned}
$$

Proof. Choose Lyapunov function as

$$
V=\frac{1}{2} S^{T} S
$$

The Lyapunov derivative corresponding to (22) is given by (26) as follows:

$$
\begin{aligned}
\dot{V}= & S^{T} \dot{S} \\
= & S^{T} G \dot{y} \\
= & S^{T}\left[G \bar{A} y(t)+G \Delta \bar{A} y(t)+G\left(\bar{A}_{d}+\Delta \bar{A}_{d}\right) y(t-\tau)\right. \\
& \left.\quad+B_{m}(u(t)+f(t))\right] .
\end{aligned}
$$

Taking into account control law (24) and Assumption 2, then

$$
\dot{V} \leq-S^{T} \Phi S<0 .
$$

The proof of Theorem 10 is complete.

Since the next section provides the design of sliding surface for multiple time-delay cases, (2) can be treated as a special case $(N=1)$. Then, it is easy to acquire the sliding surface in single time-delay condition. For brevity, the result is omitted here.

Remark 11. In accordance with state estimation in [27], when sliding function is approaching the sliding surface, the approaching exponent depends on the value of $\Phi$ in the theorem. The larger $\lambda_{\text {min }}(\Phi)$, the faster approaching speed. At the same time, an ideal sliding mode movement is difficult to acquire as it can only be converged maximally within a range surrounding the sliding surface and make back and forth through movement unceasingly.

3.3. Multiple Time-Delay System with Uncertainties. In the last section, the influence of single time-delay on system was discussed in case of internal perturbation. However, in many other cases, states of multiagent may also be influenced by multiple time-delay, and still exist a problem of parameter perturbation, as shown in (3).

By nonsingular transformation mentioned previously, Assumptions 2 to 4 are satisfied at the same time, so (3) is transformed to

$$
\begin{gathered}
\dot{y}(t)=(\bar{A}+\Delta \bar{A}(t)) y(t)+\sum_{i=1}^{N}\left(\bar{A}_{d}+\Delta \bar{A}_{d}(t)\right) y_{i}\left(t-\tau_{i}\right) \\
+\left[\begin{array}{c}
0_{(n-m) \times m} \\
B_{m}
\end{array}\right](u(t)+f(t)) \\
y(t)=\Phi(t) \quad t \in[-\tau, 0]
\end{gathered}
$$

where $\bar{A}=T A T^{-1}, \bar{A}_{d}=T A_{d} T^{-1}, \Delta \bar{A}(t)=T \Delta A(t) T^{-1}$, $\Delta \bar{A}_{d}(t)=T \Delta A_{d}(t) T^{-1}$, and $\Phi(t)=T \Psi(t)$; in addition, we have

$$
\begin{aligned}
\dot{y}_{1}(t)= & \left(\bar{A}_{11}+\Delta \bar{A}_{11}(t)\right) y_{1}(t)+\sum_{i=1}^{N}\left(\bar{A}_{d 11}+\Delta \bar{A}_{d 11}(t)\right) \\
& \times y_{i 1}\left(t-\tau_{i}\right)+\left(\bar{A}_{11}+\Delta \bar{A}_{12}(t)\right) y_{2}(t) \\
& +\sum_{i=1}^{N}\left(\bar{A}_{d 12}+\Delta \bar{A}_{d 12}(t)\right) y_{i 2}\left(t-\tau_{i}\right), \\
\dot{y}_{2}(t)= & \left(\bar{A}_{21}+\Delta \bar{A}_{21}(t)\right) y_{1}(t)+\sum_{i=1}^{N}\left(\bar{A}_{d 21}+\Delta \bar{A}_{d 21}(t)\right) \\
& \times y_{i 1}\left(t-\tau_{i}\right)+\left(\bar{A}_{22}+\Delta \bar{A}_{22}(t)\right) y_{2}(t)
\end{aligned}
$$




$$
\begin{aligned}
& +\sum_{i=1}^{N}\left(\bar{A}_{d 22}+\Delta \bar{A}_{d 22}(t)\right) y_{i 2}\left(t-\tau_{i}\right) \\
& +B_{m}(u(t)+f(t)) \\
& y_{1}(t)=\Phi_{1}(t) \quad t \in[-\tau, 0] \\
& y_{2}(t)=\Phi_{2}(t) \quad t \in[-\tau, 0]
\end{aligned}
$$

where $y_{1} \in R^{(n-m)}, y_{2} \in R^{m}, B_{m}=\sum V^{T}, \bar{A}_{11}=U_{2}^{T} A U_{2}$, $\bar{A}_{12}=U_{2}^{T} A U_{1}, \bar{A}_{d 11}=U_{2}^{T} A_{d} U_{2}, \bar{A}_{d 12}=U_{2}^{T} A_{d} U_{1}$, $\Delta \bar{A}_{11}=U_{2}^{T} G D(t) H U_{2}, \Delta \bar{A}_{d 11}=U_{2}^{T} G D(t) H_{d} U_{2}, \Delta \bar{A}_{12}=$ $U_{2}^{T} G D(t) H_{d} U_{1}, \Delta \bar{A}_{d 12}=U_{2}^{T} G D(t) H_{d} U_{1}, \Phi_{1}(t) \in R^{(n-m)}$, and $\Phi_{2}(t) \in R^{m}$ are the subblocks of $\Phi(t)$.

It is obvious that the first equation of (29) represents the dynamics of sliding motion (28), so the sliding surface is designed as

$$
S=C y_{1}+y_{2}=\left[\begin{array}{ll}
C & I
\end{array}\right] y=0
$$

where $C \in R^{m \times(n-m)}$ and $y=\left[\begin{array}{l}y_{1} \\ y_{2}\end{array}\right]$, substituting $y_{2}=-C y_{1}$ to the first equation of (29), then the sliding motion is

$$
\begin{aligned}
\dot{y}_{1}(t)= & \left(\bar{A}_{11}+\Delta \bar{A}_{11}-\bar{A}_{12} C-\Delta \bar{A}_{12} C\right) y_{1}(t) \\
& +\sum_{i=1}^{N}\left(\bar{A}_{d 11}+\Delta \bar{A}_{d 11}-\bar{A}_{d 12} C-\Delta \bar{A}_{d 12} C\right) \\
& \times y_{i 1}\left(t-\tau_{i}\right), \\
& y_{1}(t)=\Phi_{1}(t) \quad t \in[-\tau, 0] .
\end{aligned}
$$

Definition 12 (see [28]). The uncertain sliding motion (31) is said to be quadratically stabilizable if there exists symmetric positive-definite matrix $P, Q \in R^{(n-m) \times(n-m)}$, for any admissible uncertainty the derivation of the Lyapunov functional

$$
V\left(y_{1}(t), t\right)=y_{1}^{T}(t) P y_{1}(t)+\int_{t-\tau}^{t} y_{1}^{T}(s) Q y_{1}(s) d s
$$

with respect to time $t$ satisfies

$$
L\left(y_{1}(t), t\right)=\dot{V}\left(y_{1}(t), t\right) \leq 0
$$

for all pairs $\left(y_{1}(t), t\right) \in R^{(n-m)} \times R$.
For this definition, the purpose is to design a constant gain $C \in R^{m \times(n-m)}$ and a control law $u(t)$ to make the sliding motion quadratically stable and the system achieve asymptotic stabilization.

The first conclusion is about the design of sliding surface.

Theorem 13. The reduced order system (31) is quadratically stable if there exists symmetric positive-definite matrix $Z \in$ $R^{(n-m) \times(n-m)}, L \in R^{(n-m) \times n}, W \in R^{(n-m) \times n}$, and a general matrix $K \in R^{(n-m) \times(n-m)}$ as well as a positive constant $\mathcal{E}$ making (34) holds, where $\Lambda=Z \bar{A}_{11}^{T}-K^{T} \bar{A}_{12}+\bar{A}_{11} Z-$ $\bar{A}_{12} K+\varepsilon U_{2}^{T} G G^{T} U_{2}, \eta=(1 / \sqrt{N}) \bar{A}_{d 11} L-(1 / \sqrt{N}) \bar{A}_{d 12} W$, $\delta=Z U_{2}^{T} H^{T}-K U_{1}^{T} H^{T}$, and $\theta=(1 / \sqrt{N}) L U_{2}^{T} H_{d}^{T}-$ $(1 / \sqrt{N}) W U_{1}^{T} H_{d}^{T}$. Moreover, the sliding surface of (29) is $S=$ $C y_{1}(t)+y_{2}(t)=\left[\begin{array}{ll}C & I\end{array}\right] Y=0$

$$
\left[\begin{array}{cccc}
\Lambda & \eta & \delta & I \\
* & -V & \theta & 0 \\
* & * & -\varepsilon I & 0 \\
* & * & * & -V
\end{array}\right]<0
$$

where $*$ is matrix acquired based on matrix symmetry.

Proof. Take symmetric positive-definite matrix $P$ and $Q \in$ $R^{(n-m) \times(n-m)}$ to construct the following Lyapunov function:

$$
\begin{aligned}
V\left(y_{1}(t), t\right)= & y_{1}^{T}(t) P y_{1}(t) \\
& +\sum_{i=1}^{N} \int_{t-\tau_{i}}^{t} y_{1}^{T}(s) Q y_{1}(s) d s .
\end{aligned}
$$

For all $t \in[-\tau, 0], y_{1}(t) \neq 0$. Taking the derivative of (35) through (31), we have

$$
\begin{aligned}
\dot{V}\left(y_{1}(t), t\right)= & 2 y_{1}^{T}(t) P y_{1}(t)+N y_{1}^{T} Q y_{1}(t) \\
& -\sum_{i=1}^{N} y_{1}^{T}\left(t-\tau_{i}\right) Q y_{1}\left(t-\tau_{i}\right) .
\end{aligned}
$$

Letting $\widetilde{A}_{11}=\bar{A}_{11}-\bar{A}_{12} C, \Delta \widetilde{A}_{11}=\Delta \bar{A}_{11}-\Delta \bar{A}_{12} C=$ $U_{2}^{T} G D(t) H\left(U_{2}-U_{1} C\right), \widetilde{A}_{d 11}=\bar{A}_{d 11}-\bar{A}_{d 12} C, \Delta \widetilde{A}_{d 11}=\Delta \bar{A}_{d 11}-$ $\Delta \bar{A}_{d 12} C=U_{2}^{T} G D(t) H_{d}\left(U_{2}-U_{1} C\right)$, and $Y(t-\tau)=\left[\begin{array}{c}y_{1}\left(t-\tau_{1}\right) \\ y_{2}\left(t-\tau_{2}\right) \\ \vdots \\ y_{N}\left(t-\tau_{N}\right)\end{array}\right]$, so there is

$$
\begin{aligned}
\dot{V}\left(y_{1}(t), t\right)= & {\left[\begin{array}{c}
y_{1}(t) \\
y_{1}\left(t-\tau_{1}\right) \\
\vdots \\
y_{N}\left(t-\tau_{N}\right)
\end{array}\right]^{T}\left[\begin{array}{cc}
\left(\bar{A}_{11}+\Delta \widetilde{A}_{11}\right)^{T} P+P\left(\bar{A}_{11}+\Delta \widetilde{A}_{11}\right)+N Q P\left(\widetilde{A}_{d 11}+\Delta \widetilde{A}_{d 11}\right) \\
\left(\widetilde{A}_{d 11}+\Delta \widetilde{A}_{d 11}\right)^{T} P & -Q
\end{array}\right] } \\
& \times\left[\begin{array}{c}
y_{1}(t) \\
y_{1}\left(t-\tau_{1}\right) \\
\vdots \\
y_{N}\left(t-\tau_{N}\right)
\end{array}\right]
\end{aligned}
$$




$$
\begin{aligned}
= & {\left[\begin{array}{c}
y_{1}(t) \\
Y(t-\tau)
\end{array}\right]^{T}\left[\begin{array}{cc}
\left(\bar{A}_{11}+\Delta \widetilde{A}_{11}\right)^{T} P+P\left(\bar{A}_{11}+\Delta \widetilde{A}_{11}\right)+N Q & P\left(\widetilde{A}_{d 11}+\Delta \widetilde{A}_{d 11}\right) \\
\left(\widetilde{A}_{d 11}+\Delta \widetilde{A}_{d 11}\right)^{T} P & -Q
\end{array}\right] } \\
& \times\left[\begin{array}{c}
y_{1}(t) \\
Y(t-\tau)
\end{array}\right] .
\end{aligned}
$$

Letting $Z=P^{-1}$ and $J=Z Q Z$, (37) can be written as

$$
\dot{V}\left(y_{1}(t), t\right)=\left[\begin{array}{c}
P y_{1}(t) \\
P Y(t-\tau)
\end{array}\right]^{T} M\left[\begin{array}{c}
P y_{1}(t) \\
P Y(t-\tau)
\end{array}\right]
$$

where

$M$

$$
=\left[\begin{array}{cc}
Z\left(\widetilde{A}_{11}+\Delta \widetilde{A}_{11}\right)^{T}+\left(\widetilde{A}_{11}+\Delta \widetilde{A}_{11}\right) Z+N J & \left(\widetilde{A}_{d 11}+\Delta \widetilde{A}_{d 11}\right) Z \\
Z\left(\widetilde{A}_{d 11}+\Delta \widetilde{A}_{d 11}\right)^{T} & -J
\end{array}\right] .
$$

Substituting (4) to (39), it follows from Lemma 5 that

$$
\begin{aligned}
M= & {\left[\begin{array}{cc}
Z \widetilde{A}_{11}^{T}+\widetilde{A}_{11} Z+N J & \widetilde{A}_{d 11} Z \\
Z \widetilde{A}_{d 11}^{T} & -J
\end{array}\right]+\left[\begin{array}{c}
U_{2}^{T} G \\
0
\end{array}\right] } \\
& \times D(t)\left[H\left(U_{2}-U_{1} C\right) Z H_{d}\left(U_{2}-U_{1} C\right) Z\right] \\
& +\left[H\left(U_{2}-U_{1} C\right) Z H_{d}\left(U_{2}-U_{1} C\right) Z\right]^{T} \\
& \times D^{T}(t)\left[\begin{array}{c}
U_{2}^{T} G \\
0
\end{array}\right]^{T} .
\end{aligned}
$$

According to Lemma 6, $M<0$ is equivalent to

$$
\left[\begin{array}{ccc}
Z \widetilde{A}_{11}^{T}+\widetilde{A}_{11} Z+N J+\varepsilon U_{2}^{T} G G^{T} U_{2} & \widetilde{A}_{d 11} Z & Z\left(U_{2}-U_{1} C\right)^{T} H^{T} \\
Z \widetilde{A}_{d 11}^{T} & -J & Z\left(U_{2}-U_{1} C\right)^{T} H_{d}^{T} \\
H\left(U_{2}-U_{1} C\right) Z & H_{d}\left(U_{2}-U_{1} C\right) Z & -\varepsilon I
\end{array}\right]<0 .
$$

In addition, we have

$$
\left[\begin{array}{cccc}
Z \widetilde{A}_{11}^{T}+\widetilde{A}_{11} Z+\varepsilon U_{2}^{T} G G^{T} U_{2} & \widetilde{A}_{d 11} Z & Z\left(U_{2}-U_{1} C\right)^{T} H^{T} & I \\
Z \widetilde{A}_{d 11}^{T} & -J & Z\left(U_{2}-U_{1} C\right)^{T} H_{d}^{T} & 0 \\
H\left(U_{2}-U_{1} C\right) Z & H_{d}\left(U_{2}-U_{1} C\right) Z & -\varepsilon I & 0 \\
I & 0 & 0 & -(N J)^{-1}
\end{array}\right]<0
$$

Multiply $\operatorname{diag}\left\{I(1 / \sqrt{N}) J^{-1} \quad I \quad I\right\}$ on both ends of the matrix, then

$$
\left[\begin{array}{cccc}
Z \widetilde{A}_{11}^{T}+\widetilde{A}_{11} Z+\varepsilon U_{2}^{T} G G^{T} U_{2} & \frac{1}{\sqrt{N}} \widetilde{A}_{d 11} Z J^{-1} & Z\left(U_{2}-U_{1} C\right)^{T} H^{T} & I \\
\frac{1}{\sqrt{N}} J^{-1} Z \widetilde{A}_{d 11}^{T} & -(N J)^{-1} & \frac{1}{\sqrt{N}} J^{-1} Z\left(U_{2}-U_{1} C\right)^{T} H_{d}^{T} & 0 \\
H\left(U_{2}-U_{1} C\right) Z & \frac{1}{\sqrt{N}} J^{-1} H_{d}\left(U_{2}-U_{1} C\right) Z & -\varepsilon I & 0 \\
I & 0 & 0 & -(N J)^{-1}
\end{array}\right]<0 .
$$


Letting $C Z=K, Q^{-1} Z^{-1}=L, C Q^{-1} Z^{-1}=W$, and $(N J)^{-1}=V$, then (43) can be expressed as

$$
\left[\begin{array}{cccc}
\Lambda & \eta & \delta & I \\
* & -V & \theta & 0 \\
* & * & -\varepsilon I & 0 \\
* & * & * & -V
\end{array}\right]<0
$$

where $\Lambda=Z \bar{A}_{11}^{T}-K^{T} \bar{A}_{12}+\bar{A}_{11} Z-\bar{A}_{12} K+\varepsilon U_{2}^{T} G G^{T} U_{2}, \eta=$ $(1 / \sqrt{N}) \bar{A}_{d 11} L-(1 / \sqrt{N}) \bar{A}_{d 12} W, \delta=Z U_{2}^{T} H^{T}-K U_{1}^{T} H^{T}$, and $\theta=(1 / \sqrt{N}) L U_{2}^{T} H_{d}^{T}-(1 / \sqrt{N}) W U_{1}^{T} H_{d}^{T}$. So the proof of Theorem 13 is complete.

The following theorem presents the design of sliding mode controller.

Theorem 14. For a multiagent system (3), which satisfies Assumptions 2 to 4, system can be driven onto sliding surface (45) in limited time with control law (46), which means system from any states will meet the reaching condition

$$
\begin{gathered}
S=\left[\begin{array}{cc}
C & I
\end{array}\right] T x(t)=R x(t)=0, \\
u(t)=u_{e q}+u_{s w},
\end{gathered}
$$

where equivalent control $u_{e q}=-\left(B^{T} B\right)^{-1}\left[B^{T} A x(t)+\right.$ $\left.B^{T} A_{d} N X\right]$ and nonlinear switch control $u_{s w}=$ $-\left(B^{T} B\right)^{-1}\left[B^{T} G\left(H x(t)+H_{d} N X\right)+\left(B^{T} B\right) \delta_{f}+\varepsilon\right]$, in which $\varepsilon$ is a positive constant, and $\left\|x_{i}\left(t-\tau_{i}\right)\right\|_{i \in N} \leq X$.

Proof. Conside the following Lyapunov function:

$$
V(t)=\frac{1}{2} S^{T} S .
$$

Apparently, for all $S(x, t) \neq 0, V$ is positive-definite. Taking the derivative of (47) corresponding to (3), then

$$
\begin{aligned}
\dot{V}= & S^{T} \dot{S} \\
= & S^{T} R \dot{x} \\
= & S^{T} R\left[(A+\Delta A) x+\left(A_{d}+\Delta A_{d}\right)\right. \\
& \left.\quad \times \sum_{i=1}^{N} x_{i}\left(t-\tau_{i}\right)+B(u+f)\right] \\
\leq & S^{T} R A x+S^{T} R \Delta A x+S^{T} R\left(A_{d}+\Delta A_{d}\right) N X \\
& +S^{T} B_{m}(u+f) \\
\leq & S^{T} R A x+S^{T} R G H x+S^{T} R\left(A_{d}+G H_{d}\right) N X \\
& +S^{T} B_{m}(u+f) .
\end{aligned}
$$

Considering control law (46), then

$$
\begin{aligned}
\dot{V} & \leq S^{T}\left[\left(-B_{m} \delta_{f}-\varepsilon\right) \operatorname{sgn}(S)+B_{m} \delta_{f}\right] \\
& \leq-\varepsilon S^{T} \operatorname{sgn}(S) \\
& \leq 0 .
\end{aligned}
$$

This indicates that system from any states all satisfies the sliding mode reaching condition and the proof of Theorem 14 is complete.

Remark 15. Nonlinear transformation reduces system order, which simplifies the design of sliding mode control system, yet problem arising from multiple time-delay system requires reinforcement of control (46) in order to stabilize the system onto the sliding surface.

\section{Numerical Simulations}

In this section, 3 simulations are given for testing the theorems developed in this paper. Considering MAS (1), transform the case on [29], where

$$
\begin{gathered}
A=\left[\begin{array}{ccc}
2 & 0 & -1 \\
1.75 & 0.25 & 0.8 \\
1 & 0 & 1
\end{array}\right], \quad A_{d}=\left[\begin{array}{ccc}
-1 & 0 & 0 \\
-0.1 & 0.25 & 0.2 \\
-0.2 & 0.4 & 0.5
\end{array}\right], \\
f(t)=0.2 \sin (t), \quad \tau=0.5 s, \\
B=\left[\begin{array}{l}
0 \\
0 \\
1
\end{array}\right] .
\end{gathered}
$$

The initial states are $x(t)=\left[\begin{array}{lll}1 & 0.4 & -0.8\end{array}\right]^{T}$ and $t \epsilon$ $\left[\begin{array}{ll}-0.5 & 0\end{array}\right]$. Based on Theorem 8, we have

$$
\begin{gathered}
X=\left[\begin{array}{ccc}
13.7445 & -30.1667 & 50.9141 \\
-30.1667 & 103.4341 & -49.3690 \\
50.9141 & -49.3690 & 364.0445
\end{array}\right], \\
V=\left[\begin{array}{ccc}
23.0217 & 0.7174 & 34.5685 \\
0.7174 & 264.0987 & -55.7579 \\
34.5685 & -55.7579 & 350.0517
\end{array}\right], \\
L=\left[\begin{array}{lll}
-148.8728 & 263.3331 & 782.5076
\end{array}\right] .
\end{gathered}
$$

Designed to make $(A, B)$ stabilization $K$ is $K=$ $\left[\begin{array}{lll}-30.3839 & -2.9018 & 9.25\end{array}\right]$, where the poles of closed-loop are configured at $J=\left[\begin{array}{lll}-1 & -2 & -3\end{array}\right]$.

Thus, the sliding mode coefficient is acquired as

$$
P=X^{-1}=\left[\begin{array}{ccc}
0.9707 & 0.2334 & -0.1041 \\
0.2334 & 0.0665 & -0.0236 \\
-0.1041 & -0.0236 & 0.0141
\end{array}\right] \text {. }
$$

The parameter of controller (6) is $\delta_{f}=0.2$ and $\varepsilon=0.15$, then the simulation result is shown in Figure 3.

In fact, it is hard to acquire ideal sliding mode. From the figure, we can see that the practical sliding mode is converged within a region around the ideal sliding surface. 


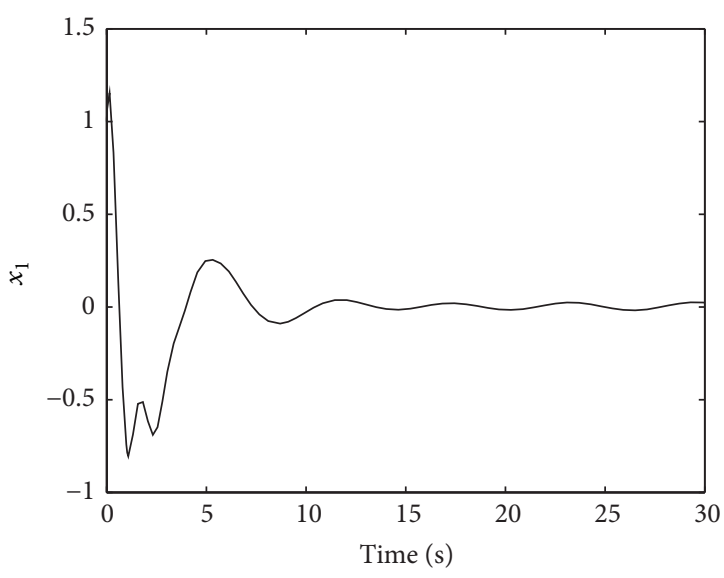

(a)

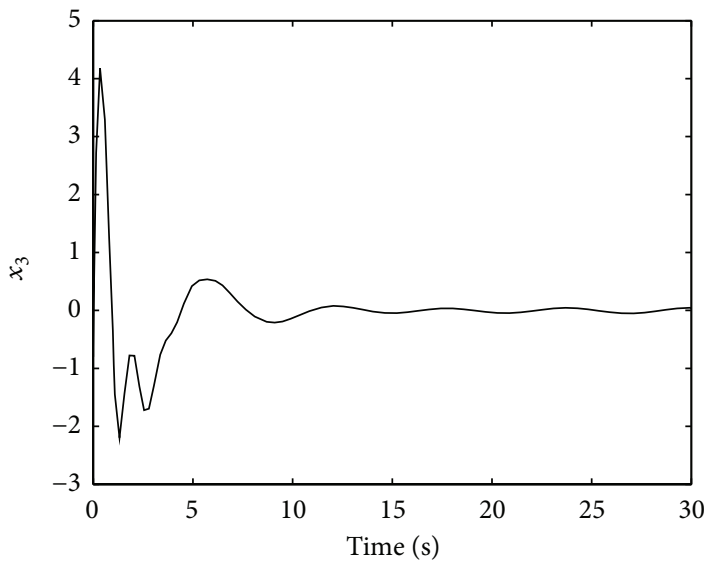

(c)

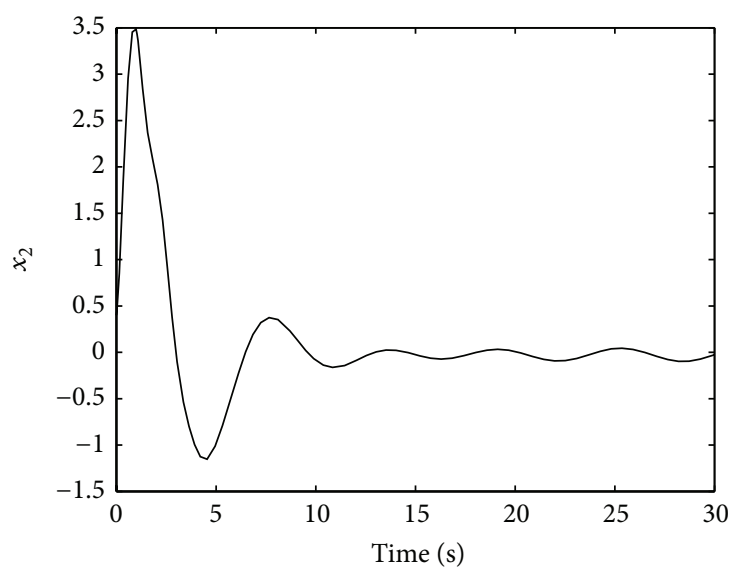

(b)

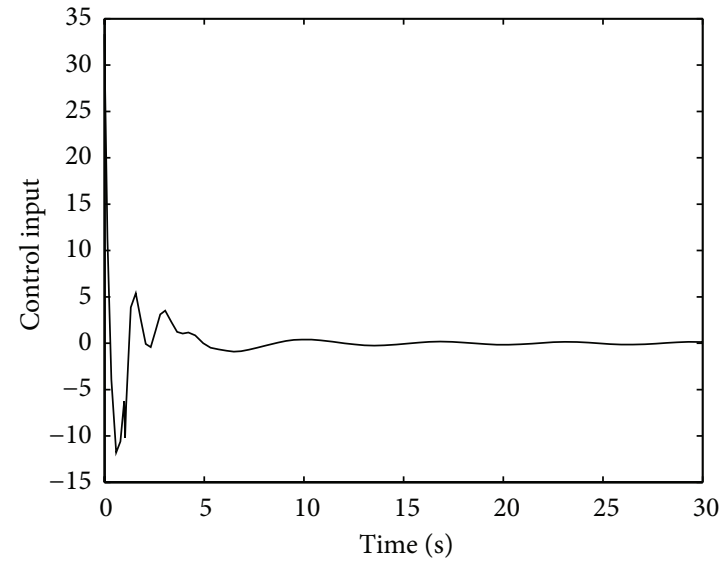

(d)

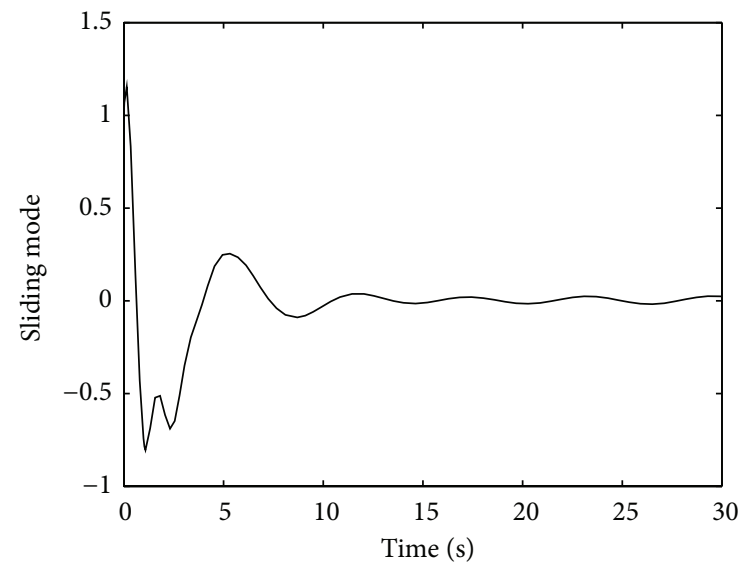

(e)

Figure 3: States $(x 1, x 2, x 3)$, control input, and sliding mode.

Considering MAS (2), where

$$
A=\left[\begin{array}{cc}
-4 & 1.5 \\
4 & 2
\end{array}\right], \quad G=\left[\begin{array}{cc}
0.1 & 0.2 \\
0 & 0.2
\end{array}\right]
$$

$D(t)=\left[\begin{array}{cc}0.5 \sin (t) & 0 \\ 0 & 0.5 \sin (t)\end{array}\right], \quad H=\left[\begin{array}{cc}0.3 & 0.2 \\ 0.2 & 0.33\end{array}\right]$,

$$
\begin{gathered}
H_{d}=\left[\begin{array}{cc}
0.27 & 0.17 \\
0.18 & 0.3
\end{array}\right], \quad B=\left[\begin{array}{l}
1 \\
0
\end{array}\right], \quad T=\left[\begin{array}{ll}
0 & 1 \\
1 & 0
\end{array}\right], \\
f(t)=0.2 \sin (t), \quad \tau=0.5 s .
\end{gathered}
$$

The initial states are $x(t)=\left[\begin{array}{ll}1.2 & -3.42\end{array}\right]^{T}$ and $t \in$ $\left[\begin{array}{ll}-0.5 & 0\end{array}\right]$, it follows from Theorem 10 that $c=3.56$. The 


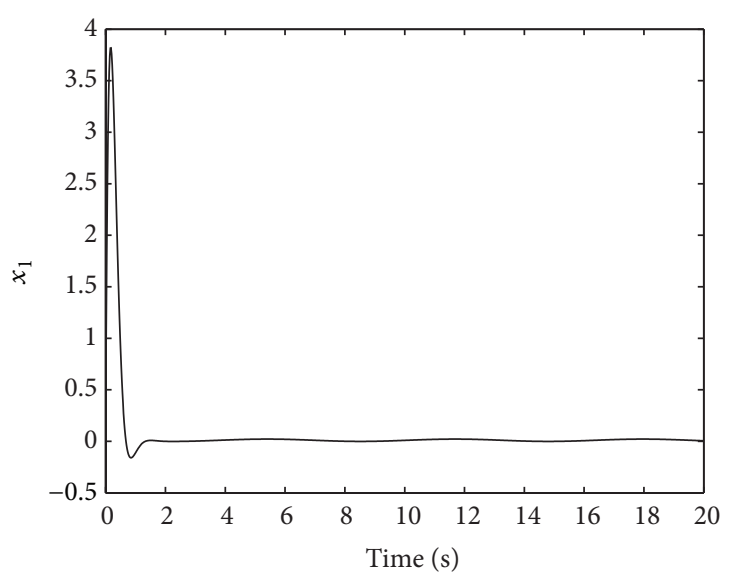

(a)

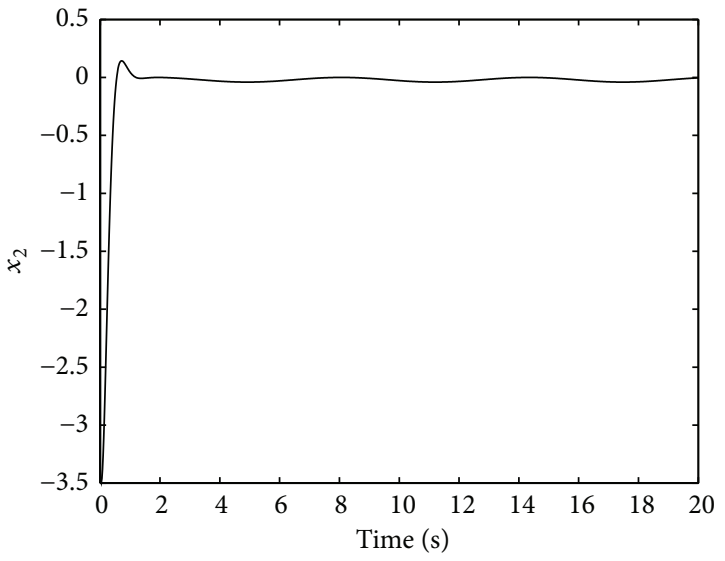

(b)

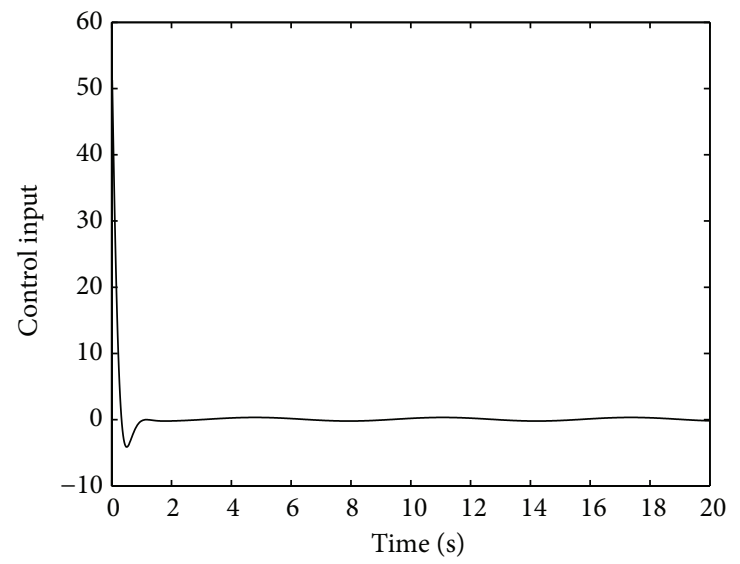

(c)

Figure 4: States $(x 1, x 2)$, and control input.

parameters of the controller are $\Phi=3.72$ and $\delta_{f}=0.2$. The simulation results are shown in Figure 4.

From the control law, although the design method is simplified, for systems requiring fast response, control parameters $\Phi$ should be enlarged.

Considering MAS (3), where

$$
\begin{gathered}
A=\left[\begin{array}{cc}
-5.2 & 2 \\
5 & 2.6
\end{array}\right], \quad G=\left[\begin{array}{cc}
0.13 & 0.3 \\
0 & 0.28
\end{array}\right], \\
D(t)=\left[\begin{array}{cc}
0.8 \sin (t) & 0 \\
0 & 0.8 \sin (t)
\end{array}\right], \quad \tau=0.5 s, \quad N=9, \\
H=\left[\begin{array}{cc}
0.4 & 0.3 \\
0.26 & 2.3
\end{array}\right], \quad H_{d}=\left[\begin{array}{cc}
0.35 & 0.22 \\
0.23 & 0.4
\end{array}\right], \\
A_{d}=\left[\begin{array}{cc}
-2.6 & 1.3 \\
2 & 1.56
\end{array}\right], \quad B=\left[\begin{array}{l}
1 \\
0
\end{array}\right], \quad f(t)=0.2 \sin (t) .
\end{gathered}
$$

The initial states are $x(t)=\left[\begin{array}{ll}2.56 & -1.42\end{array}\right]^{T}$ and $t \in$ $\left[\begin{array}{ll}-0.5 & 0\end{array}\right]$, and the nonlinear transformation is $T=\left[\begin{array}{ll}0 & 1 \\ 1 & 0\end{array}\right]$. According to Theorem 13, $Z=0.2804, L=2.5488$,
$W=7.6719$, and $K=0.8441$, the controller parameters are $\delta_{f}=0.2, \varepsilon=2.5784$, and $X=4$. The simulation results are shown in Figure 5.

\section{Conclusion}

In this paper, the sliding surface and control law of time-delay independent system without uncertainties, uncertain system with single time-delay and multiple time-delay system with uncertainties are designed. Through virtual feedback control of unstable system, poles of closed-loop are configured within the stable range. LMI is used for acquiring the parameters of sliding mode controller and getting sufficient condition for quadratic stability. Through nonsingular transformation, the equivalent reduced order system is acquired, accordingly reducing the complexity of parameter acquisition. However, it is clear that the system is eventually stabilized within an area and inevitably exists a problem of chattering. For system requiring fast response, control law could only be achieved through increasing the control parameters, yet this is technically difficult in practical engineering, and it is the subject of future work. 


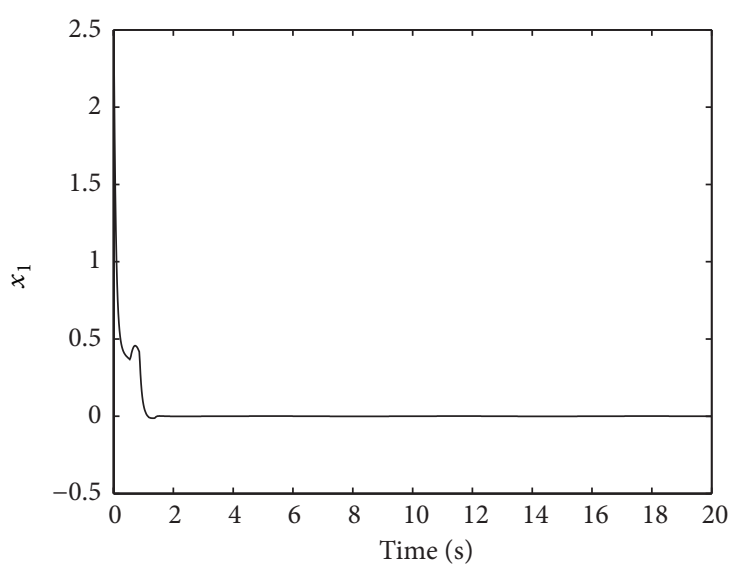

(a)

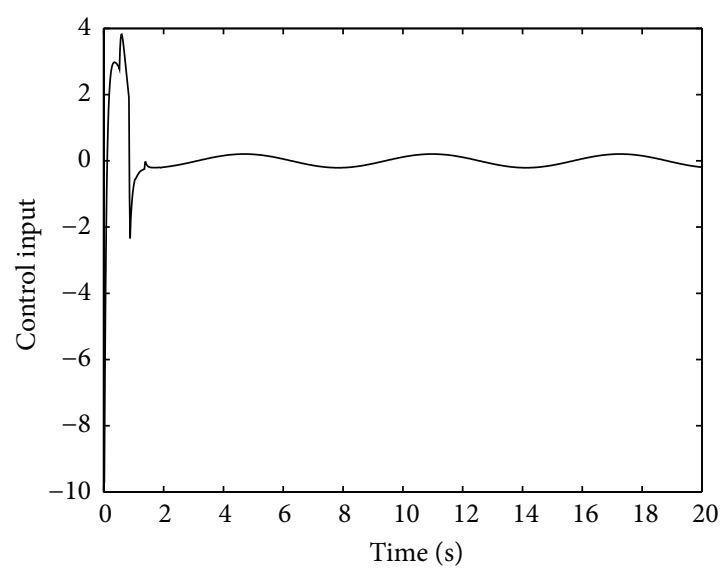

(c)

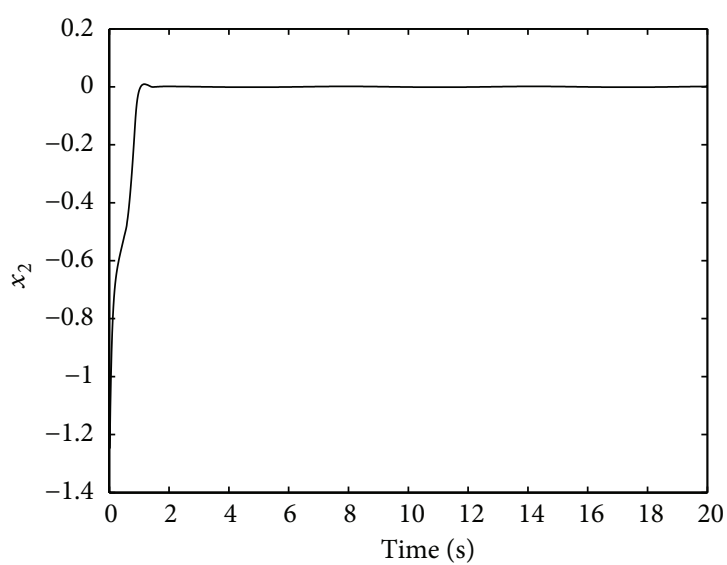

(b)

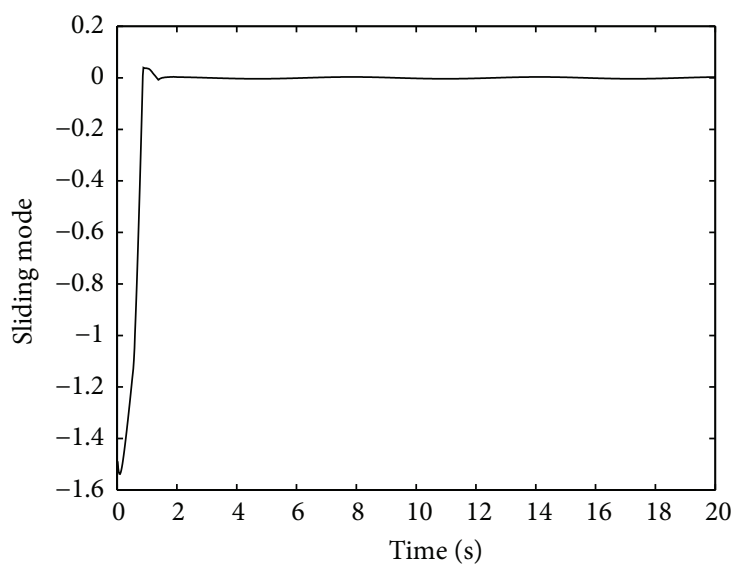

(d)

Figure 5: States $(x 1, x 2)$, control input, and sliding mode.

\section{Conflict of Interests}

The authors declare that there is no conflict of interests regarding the publication of this paper.

\section{Acknowledgments}

This work has been supported by the National Natural Science Foundation of China (Grant no. 61104109), the Natural Science Foundation of Jiangsu Province of China (Grant no. BK2011703), the Support of Science and Technology and Independent Innovation Foundation of Jiangsu Province of China (Grant no. BE2012178), and the Doctoral Fund of Ministry of Education of China (Grant no. 20113219110027).

\section{References}

[1] F. Xiao and L. Wang, "Asynchronous consensus in continuoustime multi-agent systems with switching topology and timevarying delays," IEEE Transactions on Automatic Control, vol. 53, no. 8, pp. 1804-1816, 2008.

[2] L. Wu, C. Wang, and Q. Zeng, "Observer-based sliding mode control for a class of uncertain nonlinear neutral delay systems,"
Journal of the Franklin Institute, vol. 345, no. 3, pp. 233-253, 2008.

[3] L. Wu and W. X. Zheng, "Passivity-based sliding mode control of uncertain singular time-delay systems," Automatica, vol. 45, no. 9, pp. 2120-2127, 2009.

[4] J. Hu, Z. Wang, H. Gao, and L. K. Stergioulas, "Robust sliding mode control for discrete stochastic systems with mixed time delays, randomly occurring uncertainties, and randomly occurring nonlinearities," IEEE Transactions on Industrial Electronics, vol. 59, no. 7, pp. 3008-3015, 2012.

[5] W. Su, S. V. Drakunov, and Ü. Özgüner, "Constructing discontinuity surfaces for variable structure systems: a Lyapunov approach," Automatica, vol. 32, no. 6, pp. 925-928, 1996.

[6] K. Kim, Y. Park, and S. H. Oh, "Designing robust sliding hyperplanes for parametric uncertain systems: a Riccati approach," Automatica, vol. 36, no. 7, pp. 1041-1048, 2000.

[7] C. M. Kwan, "Sliding mode control of linear systems with mismatched uncertainties," Automatica, vol. 31, no. 2, pp. 303307, 1995.

[8] L. Wu and J. Lam, "Sliding mode control of switched hybrid systems with time-varying delay," International Journal of Adaptive Control and Signal Processing, vol. 22, no. 10, pp. 909-931, 2008.

[9] H. H. Choi, "A new method for variable structure control system design: a linear matrix inequality approach," Automatica, vol. 33, no. 11, pp. 2089-2092, 1997. 
[10] L. Wu, D. W. C. Ho, and C. W. Li, "Sliding mode control of switched hybrid systems with stochastic perturbation," Systems and Control Letters, vol. 60, no. 8, pp. 531-539, 2011.

[11] H. H. Choi, "On the existence of linear sliding surfaces for a class of uncertain dynamic systems with mismatched uncertainties," Automatica, vol. 35, no. 10, pp. 1707-1715, 1999.

[12] C. W. Tao, M. Chan, and T. Lee, "Adaptive fuzzy sliding mode controller for linear systems with mismatched timevarying uncertainties," IEEE Transactions on Systems, Man, and Cybernetics B, vol. 33, no. 2, pp. 283-294, 2003.

[13] H. H. Choi, "An LMI-based switching surface design method for a class of mismatched uncertain systems," IEEE Transactions on Automatic Control, vol. 48, no. 9, pp. 1634-1638, 2003.

[14] L. Wu, H. Gao, and C. Wang, "Quasi sliding mode control of differential linear repetitive processes with unknown input disturbance," IEEE Transactions on Industrial Electronics, vol. 58, no. 7, pp. 3059-3068, 2011.

[15] L. Wu and D. W. C. Ho, "Sliding mode control of singular stochastic hybrid systems," Automatica, vol. 46, no. 4, pp. 779783, 2010.

[16] L. Wu, P. Shi, and H. Gao, "State estimation and sliding-mode control of markovian jump singular systems," IEEE Transactions on Automatic Control, vol. 55, no. 5, pp. 1213-1219, 2010.

[17] X. Li and C. E. de Souza, "Criteria for robust stability and stabilization of uncertain linear systems with state delay," Automatica, vol. 33, no. 9, pp. 1657-1662, 1997.

[18] X. Li and C. E. de Souza, "Delay-dependent robust stability and stabilization of uncertain linear delay systems: a linear matrix inequality approach," IEEE Transactions on Automatic Control, vol. 42, no. 8, pp. 1144-1148, 1997.

[19] V. L. Kharitonov and D. Melchor-Aguilar, "On delay-dependent stability conditions," Systems and Control Letters, vol. 40, no. 1, pp. 71-76, 2000.

[20] V. B. Kolmanovskii and J. P. Richard, "Stability of some linear systems with delays," IEEE Transactions on Automatic Control, vol. 44, no. 5, pp. 984-989, 1999.

[21] V. B. Kolmanovskii, "On the Liapunov-Krasovskii functionals for stability analysis of linear delay systems," International Journal of Control, vol. 72, no. 4, pp. 374-384, 1999.

[22] Y. S. Moon, P. Park, W. H. Kwon, and Y. S. Lee, "Delaydependent robust stabilization of uncertain state-delayed systems," International Journal of Control, vol. 74, no. 14, pp. 14471455, 2001.

[23] E. Fridman and U. Shaked, "Delay-dependent stability and $H_{\infty}$ control: constant and time-varying delays," International Journal of Control, vol. 76, no. 1, pp. 48-60, 2003.

[24] E. Fridman, "New Lyapunov-Krasovskii functionals for stability of linear retarded and neutral type systems," Systems and Control Letters, vol. 43, no. 4, pp. 309-319, 2001.

[25] E. Fridman and U. Shaked, "A descriptor system approach to $H_{\infty}$ control of linear time-delay systems," IEEE Transactions on Automatic Control, vol. 47, no. 2, pp. 253-270, 2002.

[26] Y. Xia and Y. Jia, "Robust sliding-mode control for uncertain time-delay systems: an LMI approach," IEEE Transactions on Automatic Control, vol. 48, no. 6, pp. 1086-1091, 2003.

[27] S. Qu, X. Wang, and Y. Wang, "A novel active sliding mode control for synchronization of uncertain chaotic systems," Kybernetes, vol. 41, no. 9, pp. 1216-1225, 2012.

[28] M. S. Mahmoud and N. F. Al-Muthairi, "Quadratic stabilization of continuous time systems with state-delay and norm-bounded time-varying uncertainties," IEEE Transactions on Automatic Control, vol. 39, no. 10, pp. 2135-2139, 1994.
[29] F. Gouaisbaut, M. Dambrine, and J. P. Richard, "Robust control of delay systems: a sliding mode control design via LMI," Systems and Control Letters, vol. 46, no. 4, pp. 219-230, 2002. 


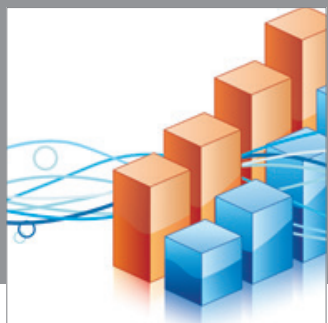

Advances in

Operations Research

mansans

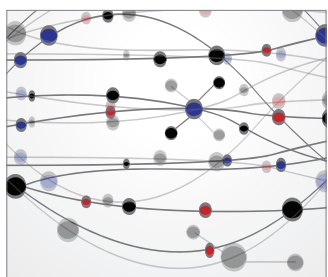

The Scientific World Journal
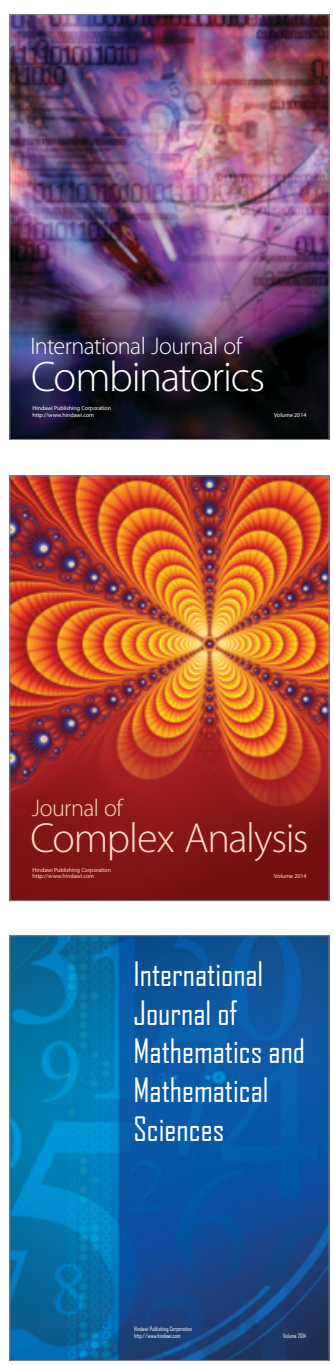
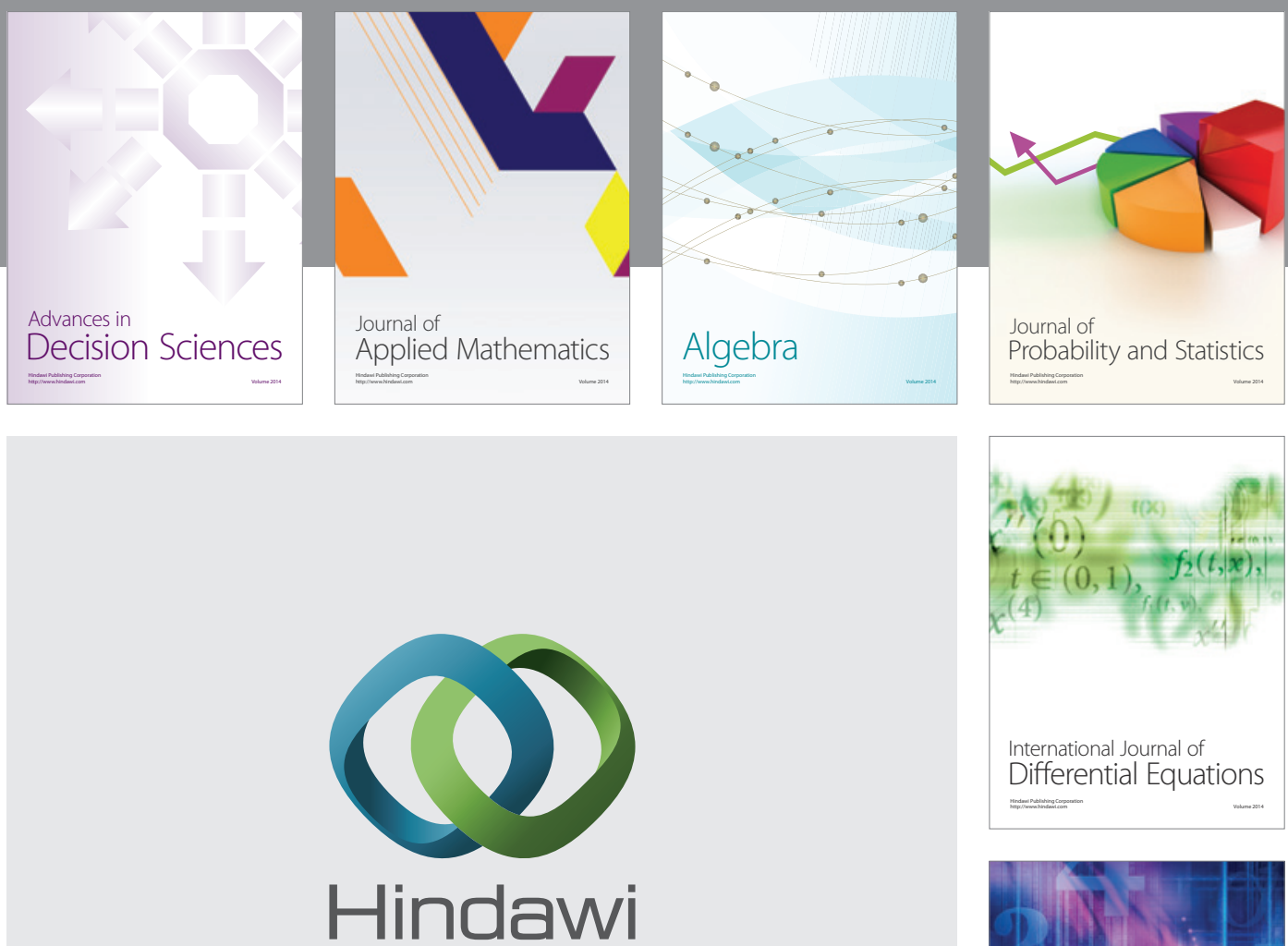

Submit your manuscripts at http://www.hindawi.com
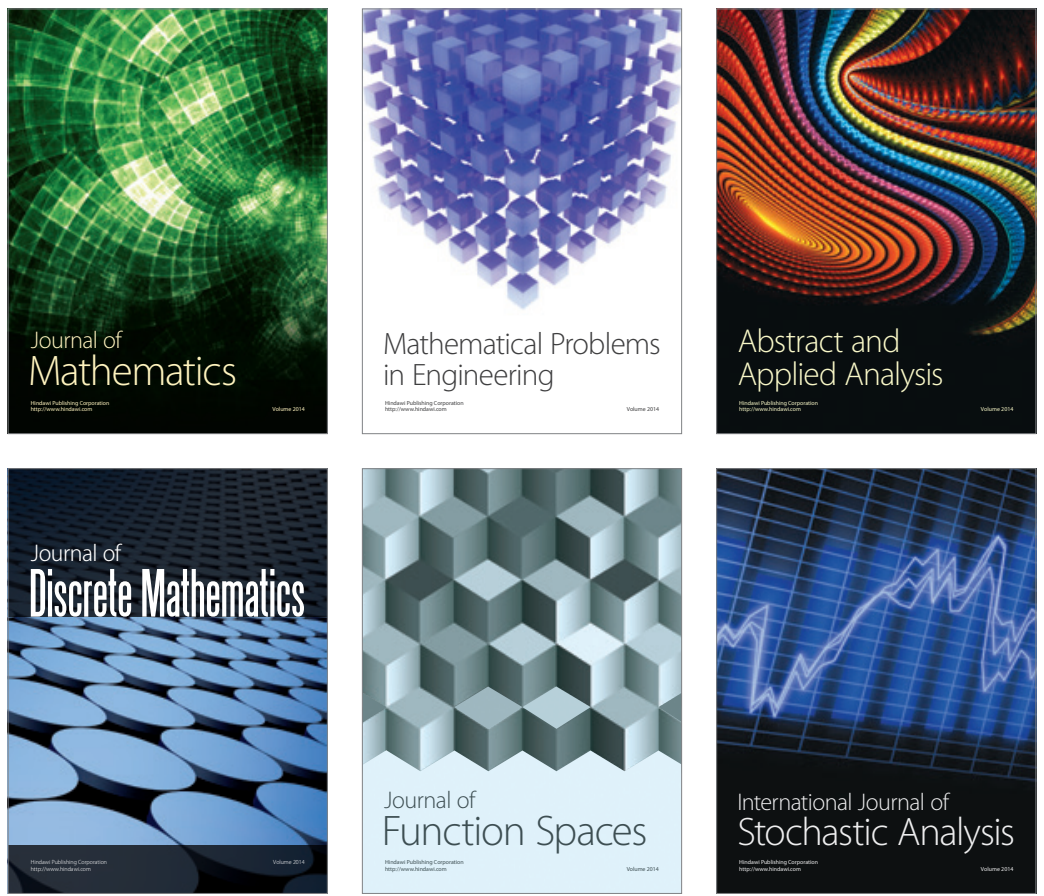

Journal of

Function Spaces

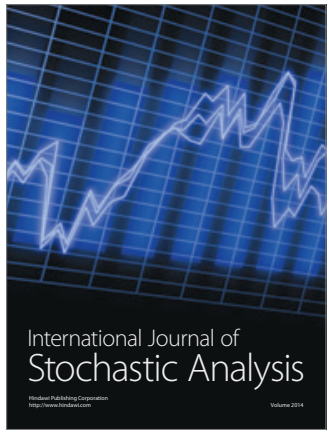

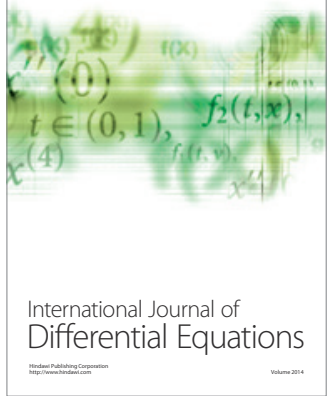
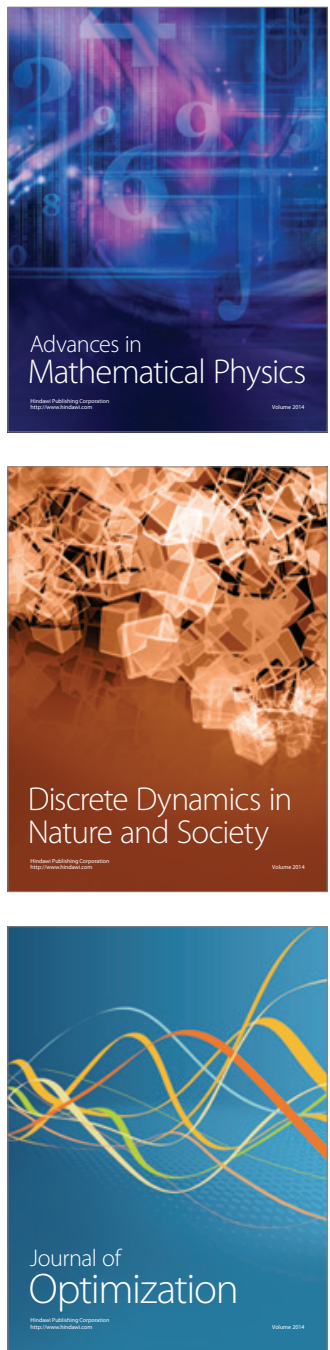